\title{
Robust convex lateral feedback control synthesis for a BWB aircraft *
}

\author{
A. Schirrer ${ }^{*}$ C. Westermayer ${ }^{*}$ M. Hemedi ${ }^{*}$ M. Kozek* \\ * Inst. of Mechanics and Mechatronics, Vienna University of \\ Technology, Wiedner Hauptstrasse 8, 1040 Vienna, Austria (e-mail: \\ alexander.schirrer@tuwien.ac.at).
}

\begin{abstract}
This paper presents the robust multi-objective design of a lateral feedback control law for a large flexible blended wing body (BWB) type passenger aircraft. An initial controller is synthesized and optimized using a convex synthesis approach based on the Youla parameterization and an LMI formulation. A multitude of both time- and frequency-domain design constraints are considered aiming to improve disturbance rejection. The obtained control law is numerically validated on several mass cases of the aircraft model, showing high loads alleviation and vibration reduction performance while obeying all stringent design constraints.
\end{abstract}

Keywords: Convex optimization; robust control; modal control; flight control; aircraft.

\section{INTRODUCTION}

Control of flexible aircraft is by now a widely studied task (Hanel [2001], Jeanneau et al. [2005], Westermayer et al. [2009], Wildschek et al. [2009]), driven by potential weight savings and thus potentially increased fuel efficiency. Novel concepts in civil aviation such as blended wing body (BWB) aircraft introduce numerous new challenges to this class of multi-objective control design problems (see Mialon and Hepperle [2005]).

The central challenge for a control engineer is to translate the given specifications efficiently and effectively into design parameters for the utilized synthesis methods (usually from optimal or robust control). Typically, these constraints are either stated as weighting functions in the frequency domain $\left(\mathcal{H}_{\infty} / \mathcal{H}_{2}\right.$ control, DK-iterations) or as objective function weightings (as in LQ control). One design method with the capability of considering both time- and frequency-domain constraints and objectives at the same time is convex synthesis, which is applied here.

Convex design for the control of conventional flexible aircraft has been studied, among others, in the $\mathrm{PhD}$ thesis of Dardenne [1999] as well as by Puyou et al. [2004] (with subsequent controller order reduction), and Torralba et al. [2009] (a self-scheduling approach).

In robust control applications, robust stability of the closed-loop is usually the most fundamental requirement. One additional, important requirement for reliable control is the stability of the controller itself (referred to as strong stabilization, see for example Ito et al. [1993] and Vidyasagar [1988]), which is not guaranteed by standard optimal and robust design methods.

This paper presents a state-of-the-art flight control design for the lateral dynamics of a large, flexible BWB transport

\footnotetext{
* This work was financially supported by EU-FP7 grant 213321. The authors are grateful for the models provided by the ACFA2020 consortium.
}

aircraft model. Numerous stringent constraints and goals are given in the time and frequency domain. An initial controller is designed using robust modal control design (Magni [2002]) to partially fulfill these goals. Based on the preshaped plant, a convex synthesis approach using the Youla parameterization and an LMI formulation is taken, optimizing directly for the remaining time- and frequencydomain goals. Moreover, a heuristic algorithm to achieve strong stabilization is proposed. A stable feedback law is obtained and validated successfully on all considered mass cases. High performance in loads alleviation and vibration reduction are achieved, and particularly the time-domain response specifications are obeyed, easing the task of subsequent command shaping (feedforward design), see Schirrer et al. [2010d].

Previous, closely related studies include a general integrated methodology for multi-objective robust control design (Schirrer et al. [2010a]), LQ-based lateral control designs of the considered BWB aircraft (Schirrer et al. [2010b]), followed by the application of a genetic algorithm for parameter optimization of a multiobjective $\mathcal{H}_{\infty}$ DKiteration design (Schirrer et al. [2010c]). The feedback controller designed in this paper is incorporated in subsequent feedforward control design in Schirrer et al. [2010d]. Longitudinal BWB control using LPV control concepts has been studied in Westermayer et al. [2010].

The paper is structured as follows: the utilized theory is listed and developed in Sec. 2, the aircraft system model is introduced in Sec. 3, followed by initial and convex feedback control design in Sec. 4 and Sec. 5. Sec. 6 discusses the obtained results, followed by conclusions.

\section{DESIGN METHODOLOGY}

A convex representation of the feedback design problem is obtained via the Youla parameterization (Youla et al. [1976]). An initial controller which performs robust pole placement via partial eigenstructure assignment is de- 


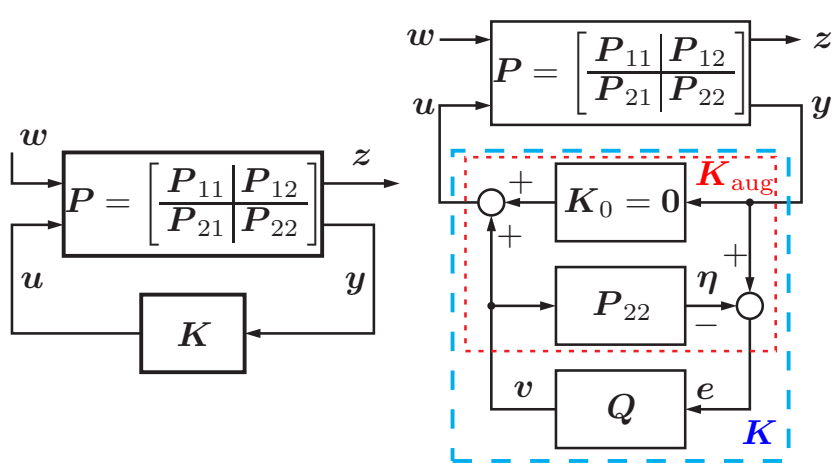

Fig. 1. Standard feedback interconnection (left), Youla parameterization (right) for stable plant $\boldsymbol{P}, \boldsymbol{K}_{0}=\mathbf{0}$

signed and absorbed into the design plant. The convex feedback synthesis is thus carried out on a chosen, preconditioned design plant in the form of an LMI problem. The obtained control law is validated and its performance is evaluated on a discrete set $\mathcal{P}=\left\{\boldsymbol{P}_{j}: j=1, \ldots, m\right\}$ of linearized validation systems (in state-space form):

$$
\dot{\boldsymbol{x}}_{j}=\boldsymbol{A}_{j} \boldsymbol{x}_{j}+\boldsymbol{B}_{j} \boldsymbol{u}_{j}, \quad \boldsymbol{y}_{j}=\boldsymbol{C}_{j} \boldsymbol{x}_{j}+\boldsymbol{D}_{j} \boldsymbol{u}_{j} .
$$

\subsection{Robust eigenstructure assignment}

As initial controller an output feedback is designed by robust eigenstructure assignment using the techniques and tools given in Magni [2002]. Given a state-space system $\boldsymbol{P}$ as in (1) (subscript $j$ omitted for brevity), $q$ triplets $\left(\lambda_{i}, \boldsymbol{v}_{i}, \boldsymbol{w}_{i}\right)$ (eigenvalue, input-, and output directions, respectively) are assigned in closed loop (with $q \leq p$ where $p$ is the number of measurements). Let $\boldsymbol{X}=\boldsymbol{C} \boldsymbol{V}+\boldsymbol{D} \boldsymbol{W}$, $\boldsymbol{V}=\left[\boldsymbol{v}_{1}, \ldots, \boldsymbol{v}_{q}\right]$, and $\boldsymbol{W}=\left[\boldsymbol{w}_{1}, \ldots, \boldsymbol{w}_{q}\right]$ hold. The output feedback gain to assign the given eigenstructure is

$$
\boldsymbol{K}=\boldsymbol{W} \boldsymbol{X}^{\dagger}
$$

where the pseudo-inverse of $\boldsymbol{X}$ yields the norm-minimal feedback gain for $q<p$, if $q=p$ and $\boldsymbol{X}$ non-singular, the inverse of $\boldsymbol{X}$ can be used instead.

\subsection{Youla parameterization}

For an appropriately partitioned plant $\boldsymbol{P}$ and a (stabilizing) feedback controller $\boldsymbol{K}$ in standard form as in Fig. 1 (left) the (stable) closed-loop transfer $\boldsymbol{T}$ is

$$
\boldsymbol{T}=\mathcal{F}_{1}(\boldsymbol{P}, \boldsymbol{K})=\boldsymbol{P}_{11}+\boldsymbol{P}_{12} \boldsymbol{K}\left(\mathbf{I}-\boldsymbol{P}_{22} \boldsymbol{K}\right)^{-1} \boldsymbol{P}_{21},
$$

where $\mathcal{F}_{1}$ is the lower LFT and $\mathbf{I}$ is a unit matrix.

The Youla parameterization adds auxiliary controller inputs $\boldsymbol{v}$ and outputs $\boldsymbol{e}$, such that the closed-loop transfer $\boldsymbol{T} \boldsymbol{e v}$ is zero. Connecting an arbitrary stable and compatible transfer function $\boldsymbol{Q}(s)$ (Youla parameter) with free order between $\boldsymbol{e}$ and $\boldsymbol{v}$ parameterizes all stabilizing controllers affinely in $\boldsymbol{Q}(s)$. In the case of an initially stable plant (or a stabilized plant with an absorbed initial controller), this extension is depicted in Fig. 1 (right), see Boyd et al. [1994]. Equation (3) becomes:

$$
\begin{array}{r}
\boldsymbol{T}=\boldsymbol{T}_{1}+\boldsymbol{T}_{2} \boldsymbol{Q} \boldsymbol{T}_{3}, \\
\boldsymbol{T}_{1}=\boldsymbol{P}_{11}, \boldsymbol{T}_{2}=\boldsymbol{P}_{12}, \boldsymbol{T}_{3}=\boldsymbol{P}_{21}, \\
\boldsymbol{Q}=\boldsymbol{K}\left(\mathbf{I}-\boldsymbol{P}_{22} \boldsymbol{K}\right)^{-1}, \\
\boldsymbol{K}=\left(\mathbf{I}+\boldsymbol{Q} \boldsymbol{P}_{22}\right)^{-1} \boldsymbol{Q} .
\end{array}
$$

With $\boldsymbol{P}_{22}$ (strictly proper) and $\boldsymbol{Q}$ (both stable),

$$
\boldsymbol{P}_{22}=\left[\begin{array}{c|c}
\boldsymbol{A}_{P} & \boldsymbol{B}_{P} \\
\hline \boldsymbol{C}_{P} & \mathbf{0}
\end{array}\right], \quad \boldsymbol{Q}=\left[\begin{array}{c|c}
\boldsymbol{A}_{Q} & \boldsymbol{B}_{Q} \\
\hline \boldsymbol{C}_{Q} & \boldsymbol{D}_{Q}
\end{array}\right],
$$

the state space representation of $\boldsymbol{K}$ is:

$$
\begin{aligned}
& \boldsymbol{x}_{K}=\left[\boldsymbol{x}_{P}^{\mathrm{T}} \mid \boldsymbol{x}_{Q}^{\mathrm{T}}\right]^{\mathrm{T}} \\
& \dot{\boldsymbol{x}}_{K}=\underbrace{\left[\begin{array}{c|c}
\boldsymbol{A}_{P}-\boldsymbol{B}_{P} \boldsymbol{D}_{Q} \boldsymbol{C}_{P} & \boldsymbol{B}_{P} \boldsymbol{C}_{Q} \\
\hline-\boldsymbol{B}_{Q} \boldsymbol{C}_{P} & \boldsymbol{A}_{Q}
\end{array}\right]}_{\boldsymbol{A}_{K}} \boldsymbol{x}_{K}+\left[\begin{array}{c}
\boldsymbol{B}_{P} \boldsymbol{D}_{Q} \\
\hline \boldsymbol{B}_{Q}
\end{array}\right] \boldsymbol{y} \\
& \boldsymbol{u}=\left[-\boldsymbol{D}_{Q} \boldsymbol{C}_{P} \mid \boldsymbol{C}_{Q}\right] \boldsymbol{x}_{K}+\boldsymbol{D}_{Q} \boldsymbol{y} .
\end{aligned}
$$

In implementations $\boldsymbol{Q}(s)$ has to be restricted to a finite, weighted sum of $n$ basis functions. A parameter-affine state-space realization $(i=1, \ldots, n)$

$$
\boldsymbol{Q}=\sum_{i=1}^{n} \boldsymbol{Q}_{i}(s) \theta_{i} \quad \boldsymbol{\theta}=\left[\theta_{1}, \ldots, \theta_{n}\right]^{\mathrm{T}}
$$

can be formed with input- or output-parametrization:

$$
\boldsymbol{Q}_{i}(s) \theta_{i}=\left[\begin{array}{c|c}
\boldsymbol{A}_{Q i} & \boldsymbol{B}_{Q i} \theta_{i} \\
\hline \boldsymbol{C}_{Q i} & \boldsymbol{D}_{Q i} \theta_{i}
\end{array}\right] \text { or }\left[\begin{array}{c|c}
\boldsymbol{A}_{Q i} & \boldsymbol{B}_{Q i} \\
\hline \theta_{i} \boldsymbol{C}_{Q i} & \theta_{i} \boldsymbol{D}_{Q i}
\end{array}\right] .
$$

\section{Remarks:}

- Stabilizing initial controllers in state observer - feedback gain form can be exploited to yield an efficient representation (see Alazard and Apkarian [1999]).

- Various choices of basis functions $\boldsymbol{Q}_{i}(s)$ in (10) are reviewed in Dardenne [1999].

\subsection{Linear Matrix Inequalities (LMIs)}

An LMI problem is a convex optimization problem commonly used in control design (see Boyd et al. [1994]) as:

$$
\begin{gathered}
\inf _{\boldsymbol{x}} \boldsymbol{c}^{\mathrm{T}} \boldsymbol{x} \\
\text { s.t. } \boldsymbol{F}(\boldsymbol{x})=\boldsymbol{F}_{0}+\sum_{i=1}^{n} x_{i} \boldsymbol{F}_{i} \succeq 0 .
\end{gathered}
$$

The matrices $\boldsymbol{F}_{i}=\boldsymbol{F}_{i}^{\mathrm{T}} \in \mathbb{R}^{n \times n}$ are symmetric and fixed, $\boldsymbol{x}=\left[x_{1}, \ldots, x_{n}\right]^{\mathrm{T}}$ are the decision variables, and $\boldsymbol{c}^{\mathrm{T}}$ is the cost vector. The constraint $\boldsymbol{F}(\boldsymbol{x}) \succeq 0$ means that the matrix $\boldsymbol{F}(\boldsymbol{x})$ be positive-semidefinite, i.e. that it possesses only non-negative eigenvalues.

\subsection{Convex control design}

With a plant parameterized in affine form (4), important time- and frequency-domain requirements can be stated as LP, QP, or LMI constraints (convex in the parameters $\boldsymbol{\theta}$ ), see Dardenne [1999]. Similarly, by bounding a constraint by an additional free variable instead of a constant, any such constraint can be turned into an objective.

Time-domain: SISO $l_{\infty}$ bounds A central benefit of convex synthesis methods is the direct incorporation of time-domain constraints and objectives, which enables template-based step-response shaping. Closed-loop timedomain responses are affine in $\boldsymbol{\theta}$ :

$$
z(t)=\mathcal{L}^{-1}\{\boldsymbol{Z}(s)\}=z_{0}(t)+\sum_{i=1}^{n} z_{i}(t) \theta_{i} .
$$

To constrain a time-domain response $z(t)$ by given lower and upper time-domain bounds $z_{\mathrm{L}}\left(t_{k}\right)<z\left(t_{k}\right)<z_{\mathrm{U}}\left(t_{k}\right)$, 
$t_{k} \in\left\{t_{1}, \ldots, t_{N}\right\}$, the expansion into the affine form yields two LP-type constraints for each $t_{k}$ :

$$
\begin{aligned}
& {\left[-z_{1}\left(t_{k}\right) \ldots-z_{n}\left(t_{k}\right)\right] \boldsymbol{\theta}<z_{0}\left(t_{k}\right)-z_{\mathrm{L}}\left(t_{k}\right),} \\
& {\left[\begin{array}{lll}
z_{1}\left(t_{k}\right) \ldots & z_{n}\left(t_{k}\right)
\end{array}\right] \boldsymbol{\theta}<z_{\mathrm{U}}\left(t_{k}\right)-z_{0}\left(t_{k}\right) .}
\end{aligned}
$$

Note that (15)-(16) also represent two (scalar) LMI constraints and can directly be plugged into an LMI problem.

Frequency-domain: MIMO $\mathcal{H}_{\infty}$ bound The constraint $\|\boldsymbol{G}\|_{\infty}<x$ can be discretized for a stable $\boldsymbol{G}(\mathrm{j} \omega)$ at a frequency grid $\omega_{k} \in\left\{\omega_{1}, \ldots, \omega_{N}\right\}$ via $N$ constraints $\bar{\sigma}\left(\boldsymbol{G}\left(\mathrm{j} \omega_{k}\right)\right)<x$. These can be translated into (real-valued) LMI constraints:

$$
\left.\left[\begin{array}{cccc}
x \mathbf{I} & \Re(\boldsymbol{G}) & \mathbf{0} & \Im(\boldsymbol{G}) \\
& x \mathbf{I} & \Im\left(\boldsymbol{G}^{\mathrm{H}}\right) & \mathbf{0} \\
& & x \mathbf{I} & \Re(\boldsymbol{G}) \\
\operatorname{sym} & & & x \mathbf{I}
\end{array}\right]\right|_{\mathrm{j} \omega_{k}} \succ 0
$$

where $\Re(\cdot), \Im(\cdot)$, and $(\cdot)^{\mathrm{H}}$ indicate the real part, imaginary part, and the Hermitian transpose, respectively. This LMI is affine in the parameters (see Dardenne [1999]). Note that this constraint asserts $\boldsymbol{G}$ stable a priori and is thus not applicable to enforce stability of the controller $\boldsymbol{K}$.

Frequency-domain: MIMO $\mathcal{H}_{2}$ bound The $\mathcal{H}_{2}$ norm of a stable, strictly proper linear dynamic system $\boldsymbol{G}(\mathrm{j} \omega)$ is

$$
\|\boldsymbol{G}(\mathrm{j} \omega)\|_{2}=h=\sqrt{\frac{1}{2 \pi} \int_{-\infty}^{\infty} \operatorname{tr}\left[(\boldsymbol{G}(\mathrm{j} \omega))^{\mathrm{H}}(\boldsymbol{G}(\mathrm{j} \omega))\right] \mathrm{d} \omega .}
$$

For a sufficiently fine and broad finite frequency gridding,

(18) can be approximated by the Riemann sum

$$
h \cong \widetilde{h}=\sqrt{\frac{1}{\pi} \sum_{k=1}^{N} \operatorname{tr}\left[\left(\boldsymbol{G}\left(\mathrm{j} \omega_{k}\right)\right)^{\mathrm{H}}\left(\boldsymbol{G}\left(\mathrm{j} \omega_{k}\right)\right)\right]\left(\omega_{k+1}-\omega_{k}\right)} .
$$

This $\mathcal{H}_{2}$ norm approximation can be expanded to a (convex) quadratic form for $\boldsymbol{G}$ affine in $\boldsymbol{\theta}(\beta \in \mathbb{R}, \gamma=$ $\left.\left[\gamma_{1}, \ldots, \gamma_{n}\right]^{\mathrm{T}} \in \mathbb{R}^{n}, \boldsymbol{\Gamma}=\left[\Gamma_{i, j}\right] \in \mathbb{R}^{n \times n}\right)$ :

$$
\begin{gathered}
\boldsymbol{G}\left(\mathrm{j} \omega_{k}\right)=\boldsymbol{G}_{k}=\widetilde{\boldsymbol{G}}_{k}+\sum_{i=1}^{n} \boldsymbol{G}_{k i} \theta_{i} \\
\widetilde{h}^{2}=\frac{1}{\pi}\left(\beta+\gamma^{\mathrm{T}} \boldsymbol{\theta}+\boldsymbol{\theta}^{\mathrm{T}} \boldsymbol{\Gamma} \boldsymbol{\theta}\right) \\
\beta=\sum_{k=1}^{N} \operatorname{tr}\left[\widetilde{\boldsymbol{G}}_{k}{ }^{\mathrm{H}} \widetilde{\boldsymbol{G}}_{k}\right], \gamma_{i}=\sum_{k=1}^{N} 2 \Re\left\{\operatorname{tr}\left[\widetilde{\boldsymbol{G}}_{k}{ }^{\mathrm{H}} \boldsymbol{G}_{k i}\right]\right\}, \\
\Gamma_{i, j}=\sum_{k=1}^{N} \Re\left\{\operatorname{tr}\left[\boldsymbol{G}_{k i}{ }^{\mathrm{H}} \boldsymbol{G}_{k j}\right]\right\} .
\end{gathered}
$$

Note that only real terms remain due to the properties of the trace and the Hermitian transpose. The matrix $\boldsymbol{\Gamma}$ is positive (semi-)definite, making the function $\widetilde{h}^{2}(\boldsymbol{\theta})$ convex, and $\boldsymbol{\Gamma}$ can be decomposed into its Cholesky factors $\boldsymbol{\Gamma}=\boldsymbol{L}^{\mathrm{T}} \boldsymbol{L}$. Then, an LMI constraint equivalent to $\tilde{h}^{2}<x\left(x \in \mathbb{R}^{+}\right)$is given as (see Sima [2001])

$$
\left[\begin{array}{c|c}
\mathbf{I}_{n} & \boldsymbol{L} \boldsymbol{\theta} \\
\hline \boldsymbol{\theta}^{\mathrm{T}} \boldsymbol{L}^{\mathrm{T}} & x-\beta-\gamma^{\mathrm{T}} \boldsymbol{\theta}
\end{array}\right] \succ 0 .
$$

Note that this constraint is of fixed size $((n+1) \times(n+1))$ with respect to the frequency grid size $N$, which allows to use high approximation precision by a fine grid in the precalculation of $\beta, \gamma$, and $\boldsymbol{\Gamma}$.

Adaptive constraint refinement The optimization problem size is primarily determined by the number of independent Youla parameter components and by the number of gridpoints at which constraints (or objectives) are defined. An adaptive grid refinement procedure has thus been implemented for high computational efficiency:

(a.1) Start with coarse design grids.

(a.2) Formulate \& solve optimization problem (12)-(13).

(a.3) Validate solution on fine validation grids.

(a.4) Pick, per violated objective/constraint, at most $n_{\text {add }}$ points that are most violated (and mutually sufficiently spaced) and add them to the design grids.

(a.5) If no violations: done. Else: return to step (2).

\subsection{Strong stabilization constraint}

To obtain a stable controller with the convex design outlined above, a constraint to ensure stability of $\boldsymbol{A}_{K}$ in (9) is necessary. The LMI formulation of Lyapunov stability $\left(\boldsymbol{P}=\boldsymbol{P}^{\mathrm{T}} \succ 0\right)$

$$
\boldsymbol{P} \boldsymbol{A}_{K}+\boldsymbol{A}_{K}{ }^{\mathrm{T}} \boldsymbol{P} \prec 0
$$

is however, although sufficient, not affine in the parameters and is thus not applicable. Moreover, high order $n_{K}$ of $\boldsymbol{A}_{K}$ yields a large problem as there are $\frac{1}{2}\left(n_{K}^{2}-n_{K}\right)$ free variables only in $\boldsymbol{P}$.

For this reason, a heuristic algorithm is proposed that embeds a convex region in the non-convex strong stabilization feasibility set $\boldsymbol{\Theta}_{\text {sstab }}=\left\{\boldsymbol{\theta} \in \mathbb{R}^{n}: \boldsymbol{\lambda}_{K}(\boldsymbol{\theta})<\mathbf{0}\right\}$. Exploiting the fact that the parameter space origin $\boldsymbol{\theta}=$ $\mathbf{0} \in \mathbf{\Theta}_{\text {sstab }}$ yields a stable controller, the algorithm is given as follows:

(b.1) Find $2 n$ simplex polytope corners $(l=1, \ldots, n$, $\boldsymbol{e}_{l}=[0, \ldots, 0,1,0, \ldots, 0]^{\mathrm{T}}$ is the $l$-th unit vector)

$$
\begin{aligned}
& \boldsymbol{\theta}_{l}^{+}=\alpha \boldsymbol{e}_{l}: \alpha=\sup _{\alpha>0} \alpha: \alpha \boldsymbol{e}_{l} \in \boldsymbol{\Theta}_{\text {sstab }} \\
& \boldsymbol{\theta}_{l}^{-}=\alpha \boldsymbol{e}_{l}: \alpha=\inf _{\alpha<0} \alpha: \alpha \boldsymbol{e}_{l} \in \boldsymbol{\Theta}_{\text {sstab }} .
\end{aligned}
$$

(b.2) Construct a hypercube $\boldsymbol{\Xi}=\left(\boldsymbol{\theta}_{1}^{-}+\ldots+\boldsymbol{\theta}_{n}^{-} \leq \boldsymbol{\theta} \leq\right.$ $\left.\boldsymbol{\theta}_{1}^{+}+\ldots+\boldsymbol{\theta}_{n}^{+}\right)$.

(b.3) Maximize a scalar $0<\delta \leq 1$ such that a given number $M \gg 1$ of randomly sampled points $\boldsymbol{\theta} \in \delta \cdot \boldsymbol{\Xi}$ pass the actual stability test $\boldsymbol{\lambda}_{K}(\boldsymbol{\theta})<\mathbf{0}$.

This construction is computationally efficient (the initial polytope has only $2 n$ vertices, the hypercube has only $2 n$ facets, and the line search over $\delta$ can be efficiently done via binary search). Finally, the inherent conservativeness can be reduced by alternately applying this algorithm and the actual LMI optimization, with the optimizer $\boldsymbol{\theta}^{*}$ as new starting point for the hypercube construction.

\section{AIRCRAFT LATERAL MODEL}

Longitudinal and lateral flight mechanics and aeroelastic effects of a large blended wing body aircraft pre-design and their coupling were modeled in an integrated fashion by the authors' project partners (Stroscher et al. [2010]). These models have been used in Westermayer et al. [2010], Schirrer et al. [2010b] and Schirrer et al. [2010c] already. 
This study only considers the lateral dynamics as well as the flexible structure modes and aerodynamic lag states to design and validate lateral control laws. A set of $k=18$ linearized state space systems (1) for various parameter values of fuel and payload mass (at fixed cruise altitude and airspeed) is available. The state vector $\boldsymbol{x}$ is composed of 4 flight-mechanic states (side slip angle $\beta$, roll rate $p$, yaw rate $r$, roll angle $\phi), 24$ elastic states (12 structural modes), as well as 10 aerodynamic lag states. The integrator states $\psi$ (yaw angle) and $y$ (horizontal displacement) are neglected in this study. These systems are augmented as shown in Fig. 2 by actuator and sensor dynamics.

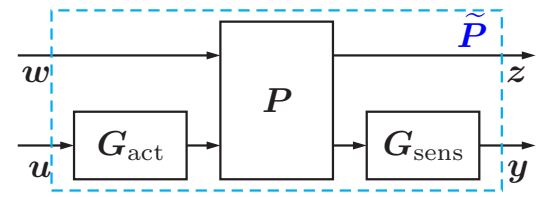

Fig. 2. Aircraft system model with actuator and sensor dynamics

Utilized inputs $\boldsymbol{u}$ for control design are:

- Symmetric rudder deflection and rate $u_{\mathrm{RU}}, \dot{u}_{\mathrm{RU}}$

- One collected antisymmetric aileron deflection and rate: outer, middle, and inner ailerons are deflected equally $\left(u_{\mathrm{AIL}}, \dot{u}_{\mathrm{AIL}}\right)$, see also Fig. 3 .

The actuator dynamics $\boldsymbol{G}_{\text {act }}$ are modeled via $2^{\text {nd }}$-order low-pass filters.

Utilized outputs $\boldsymbol{y}$ for control design are:

(1) Side slip angle $\beta_{\mathrm{CG}}$ (near the CG position)

(2) Roll rate $p$

(3) Yaw rate $r$

(4) Roll angle $\phi$

which are each subject to a time delay of $160 \mathrm{~ms}$ (due to signal processing latency, modeled via $2^{\text {nd }}$-order Padé approximations) and low-pass filtered via Butterworth filters of $2^{\text {nd }}$ order. The sensor filters are collected in $\boldsymbol{G}_{\text {sens }}$ in Fig. 2. The augmented system $\widetilde{\boldsymbol{P}}$ is of order 58 .

Additional exogenous input and output signals for validation are considered - a wind gust disturbance input (lateral wind speed $w=v_{\text {lat }}$ ) as well as two structure loads outputs $M x_{\text {wing }}$ and $M x_{\text {fin }}$ (inner moments about the x-axis at the wing root and at the fin root) and the lateral acceleration $N y_{\mathrm{CG}}$ (near the CG position).

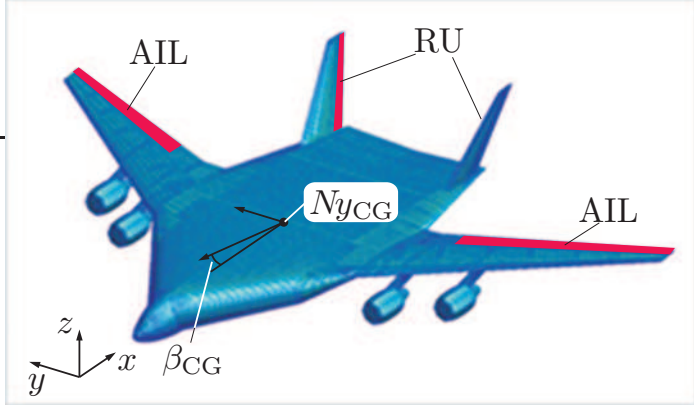

Fig. 3. BWB aircraft (RU: Rudder, AIL: Ailerons)
Table 1. Lateral control specifications

\begin{tabular}{|c|c|}
\hline \multicolumn{2}{|c|}{ General and tracking specifications } \\
\hline DR mode & $\zeta_{\mathrm{DR}} \geq 0.7, \omega_{\mathrm{DR}}$ unchanged \\
\hline Decoupling & $\begin{array}{l}\text { Generate inputs with high coupling to roll and } \\
\text { side slip angles and low cross-coupling }\end{array}$ \\
\hline Roll $\phi$ & $\begin{array}{l}\text { DC gain similar for all mass cases, rise time to } \\
90 \% \text { in } t_{\text {rise }} \leq 7 \mathrm{~s}, \max .5 \% \text { overshoot }\end{array}$ \\
\hline Side slip $\beta$ & DC gain similar for all mass cases, $t_{\text {rise }} \leq 5 \mathrm{~s}$ \\
\hline Robustness & $\begin{array}{l}\text { Stable controller, Robust Performance for all } \\
\text { mass cases }\end{array}$ \\
\hline \multicolumn{2}{|c|}{ Disturbance rejection specifications } \\
\hline \multicolumn{2}{|c|}{$\begin{array}{l}\text { Minimize the influence of lateral gust on roll, side slip, and } \\
\text { lateral acceleration, while obeying the tracking specifications } \\
\text { above. Moreover, the loads must not be increased. }\end{array}$} \\
\hline \multicolumn{2}{|c|}{ Manoeuvre loads alleviation specifications } \\
\hline
\end{tabular}

\subsection{Open-loop characteristics}

The lateral aircraft dynamics is open-loop stable for all considered mass cases at cruise condition. Modal analysis shows a prominent, low-damped Dutch Roll (DR) mode between 0.5 and $0.75 \mathrm{rad} / \mathrm{s}$ with a damping between 0.1 and 0.25 (see Fig. 4). The SISO transfer functions from rudder to side slip and to yaw rate exhibit slow SISO right half plane (RHP) zeros around $q=0.001$. These pose fundamental limitations for SISO control designs that attempt to shape side slip or yaw rate dynamics because they are far inside the required controller bandwidth. However, no slow RHP MIMO zeros appear in the MIMO plant, so MIMO designs yield satisfactory rigid-body response performance as shown in Schirrer et al. [2010b]. Moreover a number of low-damped poles affect the disturbance (lateral wind) - lateral acceleration path strongly, which is relevant for passenger comfort and possibly fatigue (see Fig. 4). The damping of the most critical mode around $20 \mathrm{rad} / \mathrm{s}$ should thus be increased robustly.

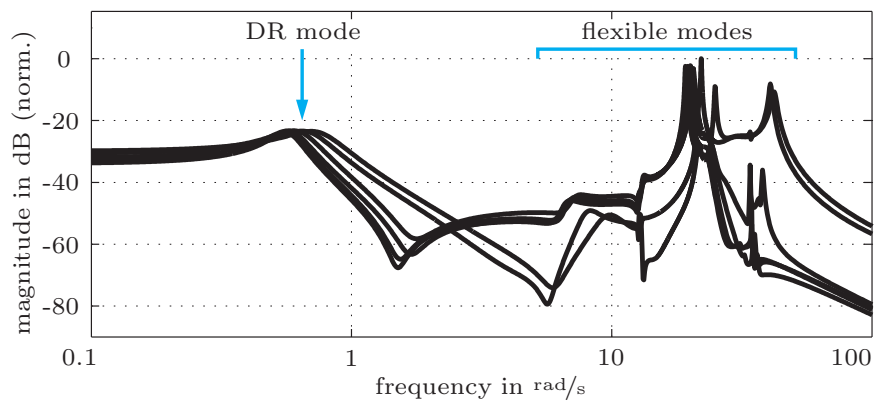

Fig. 4. Bode magnitude plot of lateral wind $v_{\text {lat }}$ - lateral acceleration $N y_{\mathrm{CG}}$ for all mass cases

\subsection{Control goals}

A control law to robustly shape the lateral reference and disturbance responses should be synthesized, with special emphasis on maneuver loads alleviation and disturbance rejection. Tab. 1 shows the set of relevant control specifications.

\section{INITIAL FEEDBACK CONTROL DESIGN}

To achieve most of the general and tracking specifications in Tab. 1, an initial controller is designed by robust partial 
eigenstructure assignment (utilizing the MATLAB Robust Modal Control Toolbox in the book Magni [2002]):

(1) The low-frequency rigid-body dynamics is robustly assigned by low-pass-filtered output feedback. A truncated design plant with rigid-body and 2nd-order actuator dynamics is utilized. The DR mode is assigned at $p_{1,2}=-0.4 \pm 0.4 \mathrm{j}$, the remaining two real poles are placed as to fulfill the step response time constants. The control law with absorbed Butterworth filters is of order 6 .

(2) The damping of a high-frequency flexible mode at about $20 \mathrm{rad} / \mathrm{s}$ is increased via a bandpass-filtered output feedback through eigenvector projection. Bandpass 2nd-order Butterworth filters are utilized, the flexible mode is reassigned at an increased damping, and a nearby mode is pinned (reassigned) at its location to avoid its destabilization. The resulting combined control law is of order 10 .

A comparison of open- and closed-loop validation step responses for selected, extremal mass cases is depicted in Fig. 5. It is evident that the controller yields mainly satisfactory results, however, the decoupling specifications are not entirely fulfilled (see roll reference (scaled aileron) to side slip). The initial control law $\boldsymbol{K}_{\text {init }}$ is absorbed into the plants, and these preconditioned plants are used for convex dynamic feedback control design.

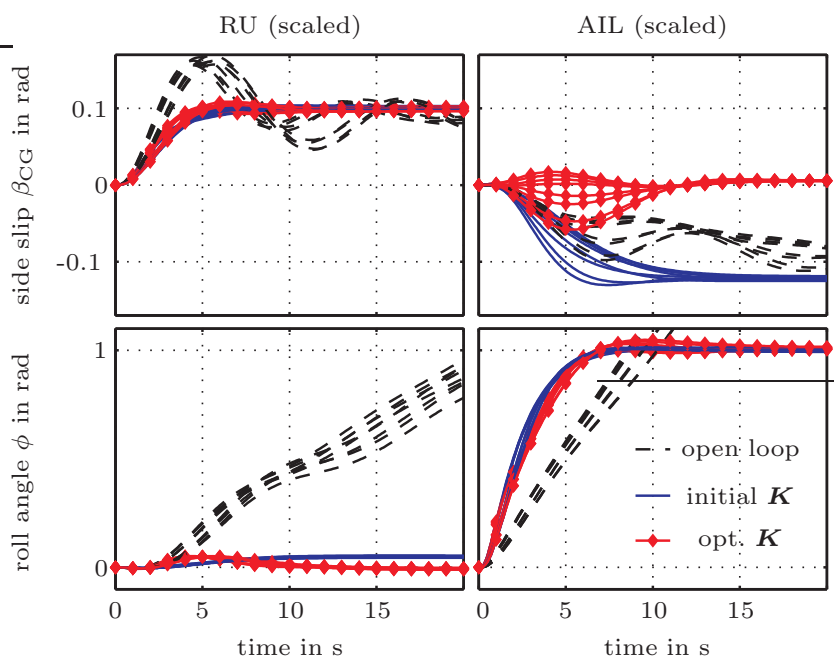

Fig. 5. Step responses of selected, extremal mass cases without controller (open-loop), with initial controller, and with the convex optimized controller

\section{CONVEX FEEDBACK CONTROLLER OPTIMIZATION}

The Youla parameterization is generated for a precompensated design plant (as outlined in Sec. 2), using an ad-hoc basis of $\boldsymbol{Q}(s)$ of total order 56 . The basis functions are simple first- and second order SISO dynamics with their poles and zeros chosen within the expected control bandwidth, for each I/O-channel. A total of 40 scalar Youla parameters (4 inputs, 2 outputs, 5 SISO basis functions each) are utilized.

The convex optimization problem is formulated with specific constraints and objectives (see Tab. 2). The cost function is the sum of an $\mathcal{H}_{\infty}$, an $\mathcal{H}_{2}$, and a time-weighted $l_{\infty}$
Table 2. Objectives (O) and constraints (C) for feedback control optimization

\begin{tabular}{ccc}
$\mathrm{O} / \mathrm{C}$ & Type \& Path & Comment \\
\hline $\mathrm{O} 1$ & min. $\mathcal{H}_{\infty}$ norm $\left(v_{\text {lat }} \rightarrow N y_{\mathrm{CG}}\right)$ & \\
$\mathrm{O} 2$ & min. $\mathcal{H}_{2}$ norm $\left(v_{\text {lat }} \rightarrow N y_{\mathrm{CG}}\right)$ & \\
$\mathrm{O} 3$ & min. $l_{\infty}$ norm $\left(\mathrm{AIL} \rightarrow N y_{\mathrm{CG}}\right)$ & \\
\hline $\mathrm{C} 1$ & $\left|\theta_{i}\right|$ bounds & stable controller \\
$\mathrm{C} 2$ & $l_{\infty}$ bounds $(\mathrm{AIL} \rightarrow$ roll $)$ & retain response \\
$\mathrm{C} 3$ & $l_{\infty}$ bounds $(\mathrm{RU} \rightarrow$ side slip) & retain response \\
$\mathrm{C} 4$ & $l_{\infty}$ bounds $(\mathrm{AIL} \rightarrow$ side slip) & decoupling \\
$\mathrm{C} 5$ & $l_{\infty}$ bounds $(\mathrm{RU} \rightarrow$ roll $)$ & decoupling \\
$\mathrm{C} 6$ & $l_{\infty}$ bounds $\left(\mathrm{AIL} \rightarrow M x_{\mathrm{fin}}\right)$ & avoid worsening \\
\hline
\end{tabular}

objective, each normalized to 1 for the initial, precompensated plant. The parameter bounds for strong stabilization were found via the algorithm in Sec. 2.5 which yields better performing stable controllers as compared to merely downscaling the Youla parameter of an unstable controller for this problem. Constraint C1 restricts the parameters to the obtained hypercube bounds.

The problem is solved to optimality and the constraint design grids are adaptively refined until all constraints are fulfilled on finely-spaced validation grids. Constraint C6 was found necessary in validation to limit fin loads in the roll maneuver, so it was added and the problem re-solved.

As an example, the $\mathrm{AIL} \rightarrow N y_{\mathrm{CG}}$ step response in Fig. 6 (right) illustrates objective $\mathrm{O} 3$ with its bounds and the adapted definition grid. This, together with constraint $\mathrm{C} 4$ (whose effect is seen in Fig. 5), has the important consequence of achieving a coordinated turn and reasonably low lateral accelerations. All other responses are at least as good as those of the precompensated plant, with a number of notable improvements after optimization:

- Decoupling and coordinated turning is realized robustly (see Fig. 5).

- Disturbance attenuation is improved, particularly in $v_{\text {lat }} \rightarrow N y_{\mathrm{CG}}$ (see Fig. 6 (left)) and $v_{\text {lat }} \rightarrow \beta_{\mathrm{CG}}$.

- Wing and fin root peak and DC loads are decreased in the RU and AIL step responses.
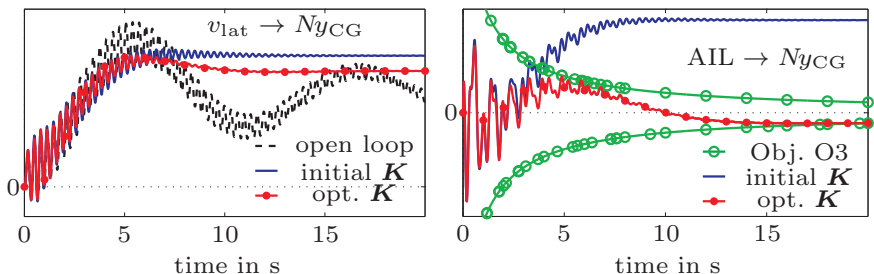

Fig. 6. Optimization results: $v_{\text {lat }} \rightarrow N y_{\mathrm{CG}}$ is reduced via frequency-domain objectives O1,O2 (left); $\mathrm{AIL} \rightarrow$ $N y_{\text {CG }}$ is reduced via a time-weighted $l_{\infty}$ objective $\mathrm{O} 3$ (right)

\section{RESULTS, ROBUSTNESS \& DISCUSSION}

As evident from Fig. 5, the final controller is robustly stable and obtains good tracking and decoupling properties for all considered mass cases (low to full fuel, low to high payload) at cruise conditions.

A high degree of disturbance rejection is achieved for $v_{\text {lat }} \rightarrow M x_{\text {fin }}, \beta_{\mathrm{CG}}, \phi$ in all cases and in $v_{\text {lat }} \rightarrow M x_{\text {wing }}$, 
$N y_{\mathrm{CG}}$ at medium and high fuel cases with typical reductions by $1 \ldots 4 \mathrm{~dB}$ in $\mathcal{H}_{2}$-norm and $5 \ldots 8 \mathrm{~dB}$ in $\mathcal{H}_{\infty}$-norm.

The final feedback controller is of dynamic order 84, which is clearly too high for implementation. For this, various robust controller order reduction methods (as discussed in Magni [2002], for example) are available.

Finally, the loads induced by the roll and side slip maneuvers are decreased as compared to the initial control design. In Schirrer et al. [2010d], the subsequent dynamic feedforward design focuses on maneuver loads alleviation specifically.

Detailed analysis of the entire validation set shows performance deficits in low-fuel mass cases for the $v_{\text {lat }} \rightarrow M x_{\text {wing }}$ and $v_{\text {lat }} \rightarrow N y_{\mathrm{CG}}$ transfers which can potentially be assessed by utilizing a robustly performing initial controller. Some suitable design methods are a robust multimodel eigenstructure assignment (see Magni [2002]), or LFTbased $\mu$ synthesis (D-G-K-iterations, Q- $\mu$-iterations Dardenne [1999]), or the synthesis of a scheduled control law (if the fuel level is available online for control) as in Torralba et al. [2009].

\section{CONCLUSIONS}

A state-of-the-art flight control design for the lateral dynamics of a large, flexible BWB transport aircraft predesign model has been performed. A multitude of partially conflicting goals and constraints in the time, frequency, and eigenstructure domains have been directly considered. An initial controller has been designed using robust partial eigenstructure assignment. Then, a convex synthesis has been performed, optimizing the closed loop for the remaining frequency- and time-domain objectives under timedomain and strong stabilization constraints. The resulting controller is validated at a set of aircraft mass cases with various fuel and payload levels at cruise conditions and performs well in the entire domain in terms of tracking, decoupling, and disturbance rejection. Problematic mass cases have been identified and discussed.

Ongoing work includes feedforward design (carried out in Schirrer et al. [2010d]), explicit incorporation of structured uncertainties (Q- $\mu$-iterations), and scheduling techniques.

\section{REFERENCES}

D. Alazard and P. Apkarian. Exact observer-based structures for arbitrary compensators. International Journal of Robust and Nonlinear Control, 9:101-118, 1999.

S. Boyd, L. El Ghaoui, E. Feron, and V. Balakrishnan. Linear Matrix Inequalities in System and Control Theory, volume 15. SIAM, 1994.

I. Dardenne. Développement de méthodologies pour la synthèse de lois de commande d'un avion de transport souple. $\mathrm{PhD}$ thesis, Ecole Natrionale Supérieure de l'Aéronautique et de l'Espace (SUPAERO), France, 1999. (title (engl.): Development of methodologies for control law synthesis for a flexible transport aircraft).

M. Hanel. Robust Integrated Flight and Aeroelastic Control System Design for a Large Transport Aircraft. Number 866 in 8. VDI-Verlag, 2001.
H. Ito, H. Ohmori, and A. Sano. Design of stable controllers attaining low $\mathcal{H}_{\infty}$ weighted sensitivity. IEEE Trans. Autom. Control, 38(3):485 - 488, 1993.

M. Jeanneau, J. Lamolie, G. Puyou, and N. Aversa. Awiators design of multi-objectives control laws. IFAC, 2005.

J.-F. Magni. Robust Modal Control with a Toolbox for Use with MATLAB. Kluwer Academic, 2002.

B. Mialon and M. Hepperle. Flying wing aerodynamics studies at ONERA and DLR. In CEAS Katnet Conference on Key Aerodynamic Technologies, Germany, 2005.

G. Puyou, G. Ferreres, C. Chiappa, and P. Menard. A multiobjective method for flight control law design. In AIAA GNC Conf., USA, 2004.

A. Schirrer, C. Westermayer, M. Hemedi, and M. Kozek. A comprehensive robust control design and optimization methodology for complex flexible-structure systems. In Proc. 18th Med. Conf. on Control \& Automation, Morocco, 2010a.

A. Schirrer, C. Westermayer, M. Hemedi, and M. Kozek. LQ-based design of the inner loop lateral control for a large flexible BWB-type aircraft. In 2010 IEEE MultiConf. on Systems and Control, Japan, 2010b.

A. Schirrer, C. Westermayer, M. Hemedi, and M. Kozek. Robust $\mathcal{H}_{\infty}$ control design parameter optimization via genetic algorithm for lateral control of a bwb type aircraft. In IFAC Workshop on Intell.Cont.Sys., RO, 2010c.

A. Schirrer, C. Westermayer, M. Hemedi, and M. Kozek. Multi-model convex design of a scheduled lateral feedforward control law for a large flexible bwb aircraft. In Proc. 18th IFAC World Congress (accepted), 2010d.

D. Sima. LMI optimization in connection with quadratic and nonlinear functions. In Proceedings of the 3rd Niconet Workshop on Numerical Software in Control and Engineering, pages 91-96, Belgium, 2001.

F. Stroscher, Ö. Petersson, and M. Leitner. Aircraft structural optimization subject to flight loads application to a wide body commercial aircraft configuration. In EASN Intl. Workshop on Aerostructures, October 2010.

J. Torralba, G. Puyou, and F. Demourant. Self-scheduling multiobjective control law design for a flexible aircraft. In AIAA GNC Conf., USA, 2009.

M. Vidyasagar. Control system synthesis: a coprime factorization approach. MIT Press, 1988.

C. Westermayer, A. Schirrer, M. Hemedi, M. Kozek, and A. Wildschek. Robust $\mathcal{H}_{\infty}$ flight and load control of a flexible aircraft using a 2DOF multi-objective design. In Proc. 2009 CACS Int. Aut. Control Conf., 2009.

C. Westermayer, A. Schirrer, M. Hemedi, and M. Kozek. Linear parameter-varying control of a large blended wing body flexible aircraft. In 18th IFAC Symposium on Automatic Control in Aerospace, Japan, 2010.

A. Wildschek, R. Maier, M. Hromcik, T. Hanis, A. Schirrer, M. Kozek, Ch. Westermayer, and M. Hemedi. Hybrid controller for gust load alleviation and ride comfort improvement using direct lift control flaps. Proc. of the 3rd EuCASS, 2009.

D.C. Youla, H.A. Jabr, and J.J. Bongiorno. Modern Wiener-Hopf design of optimal controllers. II. the multivariable case. IEEE Trans. Automat. Control, 21(3): 319-338, 1976. 